\title{
A Hybrid Subgradient Algorithm for Finding a Common Solution of an Equilibrium Problem and a Family of Strict Pseudocontraction Mappings
}

\author{
Ekkarath Thailert, Rabian Wangkeeree, and Chanoksuda Khantree \\ Department of Mathematics, Faculty of Science, Naresuan University, Phitsanulok 65000, Thailand \\ Correspondence should be addressed to Rabian Wangkeeree; rabianw@nu.ac.th
}

Received 8 June 2014; Accepted 25 July 2014; Published 20 August 2014

Academic Editor: Giuseppe Marino

Copyright (C) 2014 Ekkarath Thailert et al. This is an open access article distributed under the Creative Commons Attribution License, which permits unrestricted use, distribution, and reproduction in any medium, provided the original work is properly cited.

\begin{abstract}
We propose a new strongly convergent algorithm for finding a common point in the solution set of a class of pseudomonotone equilibrium problems and the set of common fixed points of a family of strict pseudocontraction mappings in a real Hilbert space. The strong convergence theorem of proposed algorithms is investigated without the Lipschitz condition for the bifunctions. Our results complement many known recent results in the literature.
\end{abstract}

\section{Introduction}

Let $\mathscr{H}$ be a real Hilbert space endowed with an inner product $\langle\cdot\rangle$ and a norm $\|\cdot\|$ associated with this inner product, respectively. Let $C$ be a nonempty closed convex subset of $\mathscr{H}$, and let $f$ be a bifunction from $C \times C$ to $\mathbb{R}$ such that $f(x, x)=0$ for all $x \in C$. An equilibrium problem in the sense of Blum and Oettli [1] is stated as follows:

$$
\text { Find } x^{*} \in C \text { such that } f\left(x^{*}, y\right) \geq 0 \quad \forall y \in C .
$$

Problem of the form (1) on one hand covers many important problems in optimization as well as in nonlinear analysis such as (generalized) variational inequality, nonlinear complementary problem, and nonlinear optimization problem, just to name a few. Convex minimization problems have a great impact and influence on the development of almost all branches of pure and applied sciences. On the other hand, it is rather convenient for reformulating many practical problems in economics, transportation, and engineering (see $[1,2]$ and the references quoted therein). We denote the set of solutions of the problem (1) by $\operatorname{Sol}(f, C)$.

The existence of solution and its characterizations can be found, for example, in [3], while the methods for solving problem (1) have been developed by many researchers [4, 5]. On the other hand, iterative methods for nonexpansive mappings have recently been applied to solve convex minimization problems [6]. The problem $P(C, f, S)$ of finding a common point in the solution set of problem $E P(C, f)$ and the set of fixed points of a nonexpansive mapping $S$ recently becomes an attractive subject, and various methods have been developed for solving this problem. Most of the existing algorithms for this problem are based on the proximal point method applying to equilibrium problem $E P(C, f)$ combining with a Mann's iteration to the problem of finding a fixed point of $S$.

In 2006, S. Takahashi and W. Takahashi [7] proposed an iterative scheme under the name viscosity approximation methods for finding a common element of set of solutions of (1) and the set of fixed points of nonexpansive mapping $S$ in a real Hilbert space $\mathscr{H}$. This method generated an iteration sequence $\left\{x^{k}\right\}$ starting from a given initial point $x^{0} \in \mathscr{H}$ and computed $x^{k+1}$ as

Find $u^{k} \in C$ such that

$$
\begin{gathered}
f\left(u^{k}, y\right)+\frac{1}{r_{k}}\left\langle y-u^{k}, u^{k}-x^{k}\right\rangle \geq 0, \quad \forall y \in C, \\
\text { Compute } x^{k+1}=\alpha_{k} g\left(x^{k}\right)+\left(1-\alpha_{k}\right) S\left(u^{k}\right), \quad k \geq 0,
\end{gathered}
$$


where $g$ is a contraction of $\mathscr{H}$ into itself and the sequences of parameters $\left\{r_{k}\right\}$ and $\left\{\alpha_{k}\right\}$ were chosen appropriately. Under certain choice of $\left\{\alpha_{k}\right\}$ and $\left\{r_{k}\right\}$, the authors showed that two iterative sequences $\left\{x^{k}\right\}$ and $\left\{u^{k}\right\}$ converge strongly to $z=$ $P_{\text {Fix }(S, C) \cap S o l}(f, C)(g(z))$, where $P_{C}$ denotes the projection onto C.

Recently, Anh in [8] proposed to use the extragradienttype iteration instead of the proximal point iteration given in [9] for solving problem $P(C, f, T)$. More precisely, given $z^{k} \in C$, the proximal point iteration given in [9] is replaced by the two following mathematical programs, which seems numerically easier than previous ones. More precisely, the following algorithm is suggested in [8]:

$$
\begin{gathered}
\text { For an initial point } x^{0} \in C, \\
y^{k}=\operatorname{argmin}\left\{f\left(x^{k}, y\right)+\frac{1}{2 \lambda_{k}}\left\|y-x^{k}\right\|^{2}: y \in C\right\}, \\
z^{k}=\operatorname{argmin}\left\{f\left(y^{k}, z\right)+\frac{1}{2 \lambda_{k}}\left\|z-x^{k}\right\|^{2}: z \in C\right\}, \quad k \geq 0 .
\end{gathered}
$$

It was proved that if $f$ is pseudomonotone and satisfies the Lipschitz-type condition, there are Lipschitz constants $c_{1}>0$ and $c_{2}>0$ if

$$
\begin{array}{r}
f(x, y)+f(y, z) \geq f(x, z)-c_{1}\|x-y\|^{2}-c_{2}\|y-z\|^{2}, \\
\forall x, y, z \in C,
\end{array}
$$

then the sequence $\left\{z^{k}\right\}$ strongly converges to a solution of problem $P(C, f, T)$. Recently, Anh and Muu [10] emphasized that the Lipschitz-type condition (4), in general, is not satisfied, and if yes, finding the constants $c_{1}$ and $c_{2}$ is not an easy task. Furthermore, solving the strongly convex programs (3) is expensive except special cases when $C$ has a simple structure. They suggested and studied a new algorithm for finding a common point in the solution set of a class of pseudomonotone equilibrium problems and the set of fixed points of nonexpansive mappings in a real Hilbert space. The proposed algorithm uses only one projection and does not require any Lipschitz condition for the bifunctions. More precisely, they introduced the following algorithm:

$$
\begin{gathered}
\text { Pick any } x^{0} \in C ; \\
y^{k} \in \partial_{\varepsilon_{k}} f\left(x^{k}, \cdot\right)\left(x^{k}\right) ; \\
\gamma_{k}:=\max \left\{\lambda_{k},\left\|y^{k}\right\|\right\}, \quad \alpha_{k}:=\frac{\beta_{k}}{\gamma_{k}} ; \\
w^{k}=P_{C}\left(x^{k}-\alpha_{k} y^{k}\right) ; \\
x^{k+1}=\delta_{k} w^{k}+\left(1-\delta_{k}\right) T w^{k}, \quad \text { for each } k=0,1, \ldots,
\end{gathered}
$$

where $\partial_{\varepsilon} f(x, \cdot)(x)$ stands for $\varepsilon$-subdifferential of the convex function $f(x, \cdot)$ at $x$ and $\left\{\varepsilon_{k}\right\},\left\{\lambda_{k}\right\},\left\{\beta_{k}\right\}$, and $\left\{\delta_{k}\right\}$ were chosen appropriately. Under the certain conditions, $\left\{x^{k}\right\}$ converse strongly to a common point in the solution set of a class of pseudomonotone equilibrium problems and the set of fixed points of nonexpansive mappings in a real Hilbert space.

On the other hand, the problem of finding a common fixed point element of a finite family of self-mappings $S:=$ $\left\{S_{i}\right\}_{i=1}^{p}(p \geq 1)$ is expressed as follows:

$$
\text { Find } x^{*} \in C \quad \text { such that } x^{*} \in \bigcap_{i=1}^{p} \operatorname{Fix}\left(S_{i}, C\right) \text {, }
$$

where $\operatorname{Fix}\left(S_{i}, C\right)$ is the set of the fixed points of the mapping $S_{i}(i=1, \ldots, p)$. Let us denote by

$$
\operatorname{Fix}(S, C):=\bigcap_{i=1}^{p} \operatorname{Fix}\left(S_{i}, C\right)
$$

the solution sets of the fixed-point problem (6). Problem of finding a fixed point of a mapping or a family of mappings is a classical problem in nonlinear analysis. The theory and solution methods of this problem can be found in many research papers and monographs (see [11]). The problem of finding a common fixed point of a finite sequence of mappings has been studied by many researchers. For instance, in 2005, Blum and Oettli [1] proposed an iterative algorithm for finding a common fixed point of $p$ strict pseudocontraction mapping $S_{i}(i=1, \ldots, p)$. The method computed a sequence $\left\{x^{k}\right\}$ starting from $x^{0} \in \mathscr{H}$ and taking

$$
x^{k+1}=\alpha_{k} x^{k}+\left(1-\alpha_{k}\right) \sum_{i=1}^{p} \lambda_{k, i} S_{i}\left(x^{k}\right), \quad k \geq 1,
$$

where the sequence of parameters $\left\{\lambda_{k}\right\}$ was chosen in a specific way to ensure the convergence of the iterative sequence $\left\{x^{k}\right\}$. The authors proved that the sequence $\left\{x^{k}\right\}$ converges weakly to a point $\bar{x} \in \bigcap_{i=1}^{p} \operatorname{Fix}\left(S_{i}, C\right)$. Very recently, Anh et al. [12] suggested and analyzed an algorithm for finding a common solution of two problems (1) and (6). Typically, this problem can be written as follows:

$$
\text { Find } x^{*} \in \operatorname{Fix}(S, C) \cap \operatorname{Sol}(f, C) \text {. }
$$

They presented an algorithm for finding a solution of problem (9). More precisely, they suggested the following algorithm:

$$
\text { For an initial point } x^{0} \in C \text {, }
$$

$$
\begin{gathered}
y^{k}=\operatorname{argmin}\left\{\lambda_{k} f\left(x^{k}, y\right)+\frac{1}{2}\left\|y-x^{k}\right\|^{2}: y \in C\right\}, \\
t^{k}=\operatorname{argmin}\left\{\lambda_{k} f\left(y^{k}, y\right)+\frac{1}{2}\left\|y-x^{k}\right\|^{2}: y \in C\right\}, \\
x^{k+1}=\alpha_{k} t^{k}+\left(1-\alpha_{k}\right) \sum_{i=1}^{p} \lambda_{k, i} S_{i}\left(t^{k}\right), \quad k \geq 0,
\end{gathered}
$$

where $f$ is pseudomonotone and continuous on $C$ and Lipschitz-type continuous on $C,\left\{\lambda_{k}\right\},\left\{\lambda_{k, i}\right\}$, for all $i=$ $1,2, \ldots, p$, and $\left\{\alpha_{k}\right\}$ are the sequences of parameters which 
were chosen appropriately. Under certain conditions on the parameters and $f$, they proved that a sequence $\left\{x^{k}\right\}$ converges weakly to a solution $x^{*}$ of (9).

In this paper, motivated by the idea of Anh and Muu [10] and Anh et al. [12], we propose a new algorithm for finding a common point in the solution set of a class of pseudomonotone equilibrium problems and the set of common fixed points of a family of strict pseudocontraction mappings in a real Hilbert space. The strong convergence of proposed algorithms is investigated under certain assumptions. Our results complement many known recent results in the literature.

\section{Preliminaries}

Let $C$ be a nonempty convex subset of a Hilbert space $\mathscr{H}$. We write $x^{k}-x$ to indicate that the sequence $\left\{x^{k}\right\}$ converges weakly to $x$ as $k \rightarrow \infty$ and $x^{k} \rightarrow x$ to indicate that the sequence $\left\{x^{k}\right\}$ converges strongly to $x$ as $k \rightarrow \infty$. Since $C$ is closed, convex, for any $x \in \mathscr{H}$, there exists a unique point in $C$, denoted by $P_{C}(x)$ satisfying

$$
\left\|x-P_{C}(x)\right\| \leq\|x-y\|, \quad \forall y \in C .
$$

$P_{C}$ is called the metric projection of $\mathscr{H}$ to $C$. It is well known that $P_{C}$ satisfies the following properties:

$$
\begin{aligned}
&\left\langle x-y, P_{C}(x)-P_{C}(y)\right\rangle \\
& \geq\left\|P_{C}(x)-P_{C}(y)\right\|^{2}, \quad \forall x, y \in \mathscr{H}, \\
&\left\langle x-P_{C}(x), P_{C}(x)-y\right\rangle \geq 0, \quad \forall x \in \mathscr{H}, y \in C, \\
&\|x-y\|^{2} \geq\left\|x-P_{C}(x)\right\|^{2}+\left\|y-P_{C}(x)\right\|^{2}, \\
& \forall x \in \mathscr{H}, y \in C .
\end{aligned}
$$

The concept of strict pseudocontraction is considered in [13], defined as follows.

Definition 1. Let $C$ be a nonempty closed convex subset of a real Hilbert space $\mathscr{H}$. A mapping $S: C \rightarrow C$ is said to be a strict pseudocontraction if there exists a constant $0 \leq L<1$ such that

$$
\begin{array}{r}
\|S(x)-S(y)\|^{2} \\
\leq\|x-y\|^{2}+L\|(I-S)(x)-(I-S)(y)\|^{2}, \\
\forall x, y \in C,
\end{array}
$$

where $I$ is the identity mapping on $\mathscr{H}$. If $L=0$, then $S$ is called nonexpansive on $C$.

The following proposition lists some useful properties of a strict pseudocontraction mapping.

Proposition 2 (see [13]). Let $C$ be a nonempty closed convex subset of a real Hilbert space $\mathscr{H}$; let $S: C \rightarrow C$ be a L-strict pseudocontraction, and, for each $i=1, \ldots, p, S_{i}: C \rightarrow C$ is a $L_{i}$-strict pseudocontraction for some $0 \leq L_{i}<1$. Then, (i) S satisfies the following Lipschitz condition:

$$
\|S(x)-S(y)\| \leq \frac{1+L}{1-L}\|x-y\|, \quad \forall x, y \in C ;
$$

(ii) $I-S$ is demiclosed at 0 . That is, if the sequence $\left\{x^{k}\right\}$ contains in $C$ such that $x^{k}-\bar{x}$ and $(I-S)\left(x^{k}\right) \rightarrow 0$, then $(I-S)(\bar{x})=0$;

(iii) the set of fixed points $\operatorname{Fix}(S)$ is closed and convex;

(iv) if $\eta_{i}>0(i=1, \ldots, p)$ and $\sum_{i=1}^{p} \eta_{i}=1$, then $\sum_{i=1}^{p} \eta_{i} S_{i}$ is a $\bar{L}$-strict pseudocontraction with $\bar{L}:=\max \left\{L_{i}: 1 \leq\right.$ $i \leq p\}$

(v) if $\eta_{i}$ is chosen as in (iv) and $\left\{S_{i}: i=1, \ldots, p\right\}$ has a common fixed point, then

$$
\operatorname{Fix}\left(\sum_{i=1}^{p} \eta_{i} S_{i}\right)=\bigcap_{i=1}^{p} \operatorname{Fix}\left(S_{i}, C\right) .
$$

Lemma 3 (see [14]). Suppose that $\left\{\alpha_{k}\right\}$ and $\left\{\beta_{k}\right\}$ are two sequences of nonnegative real numbers such that

$$
\alpha_{k+1} \leq \alpha_{k}+\beta_{k}, \quad k \geq 0,
$$

where $\sum_{k=0}^{\infty} \beta_{k}<\infty$. Then, the sequence $\left\{\alpha_{k}\right\}$ is convergent.

The following idea of the $\varepsilon$-subdifferential of convex functions can be found in the work of Brøndsted and Rockafellar [15] but the theory of $\varepsilon$-subdifferential calculus was given by Hiriart-Urruty [16].

Definition 4. Consider a proper convex function $\phi: C \rightarrow \overline{\mathbb{R}}$. For a given $\varepsilon>0$, the $\varepsilon$-subdifferential of $\phi$ at $x_{0} \in \operatorname{dom} \phi$ is given by

$$
\begin{aligned}
& \partial_{\varepsilon} \phi\left(x_{0}\right) \\
& \quad=\left\{x \in C: \phi(y)-\phi\left(x_{0}\right) \geq\left\langle x, y-x_{0}\right\rangle-\varepsilon, \forall y \in C\right\} .
\end{aligned}
$$

Remark 5. It is known that if the function $\phi$ is proper lower semicontinuous convex, then, for every $x \in \operatorname{dom} \phi$, the $\varepsilon$ subdifferential $\partial_{\varepsilon} \phi(x)$ is a nonempty closed convex set (see [17]).

\section{Main Results}

Now, we are in a position to state and prove the main strong convergence theorem for the given iterative scheme.

Assumption 6. Let the bifunction $f: C \times C \rightarrow \mathbb{R}$ be satisfied by the following conditions:

(i) for each $x, f(x, x)=0$ and $f(x, \cdot)$ is lower semicontinuous convex on $C$;

(ii) if $\left\{x^{k}\right\} \subseteq C$ is bounded and $\varepsilon_{k} \downarrow 0$ as $k \rightarrow \infty$, then the sequence $\left\{y^{k}\right\}$ with $y^{k} \in \partial_{\varepsilon_{k}} f\left(x^{k}, \cdot\right)\left(x^{k}\right)$ is bounded; 
(iii) $f$ is pseudomonotone on $C$ with respect to every solution of $E P(C, f)$ and satisfies the following condition, called strict paramonotonicity property:

$x \in \operatorname{Sol}(C, f), y \in C, f(y, x)=0 \Longrightarrow y \in \operatorname{Sol}(C, f)$;

(iv) for each $x \in C, f(\cdot, x)$ is weakly upper semicontinuous on the open set $C$.

Assumption 7. Consider the following:

(i) for each $i=1,2, \ldots, p, S_{i}$ is $L_{i}$-strict pseudocontraction for some $0 \leq L_{i}<1$;

(ii) the solution set $\Omega$ of the problem $P\left(C, f, S_{i}\right)$ is nonempty; that is,

$$
\Omega:=\operatorname{Sol}(f, C) \bigcap \bigcap_{i=1}^{p} \operatorname{Fix}\left(S_{i}, C\right) \neq \emptyset .
$$

Assumption 8. Suppose that the sequences $\left\{\lambda_{k}\right\},\left\{\beta_{k}\right\},\left\{\varepsilon_{k}\right\}$, $\left\{\eta_{k, i}\right\}$, and $\left\{\delta_{k}\right\}$ of nonnegative numbers satisfy the following conditions:

(i) $0<\lambda_{k}<\lambda, 0<\bar{L}<\delta_{k}<1$, and $\delta_{k} \rightarrow 1 / 2$, where $\bar{L}:=\max \left\{L_{i}: 1 \leq i \leq p\right\}$

(ii) $\beta_{k}>0, \sum_{k=0}^{\infty} \beta_{k}=+\infty$, and $\sum_{k=0}^{\infty} \beta_{k}^{2}<+\infty$;

(iii) $\sum_{k=0}^{\infty} \beta_{k} \varepsilon_{k}<+\infty$;

(iv) $\sum_{i=1}^{p} \eta_{k, i}=1$ for all $k \geq 1$.

Algorithm 9. Now, the iteration scheme for finding a common point in the set of solutions of problem $E P(C, f)$ and the set of common fixed points of $L_{i}$-strict pseudocontraction, for each $i=1,2, \ldots, p$, can be written as follows:

$$
\begin{gathered}
x^{0} \in C ; \\
y^{k} \in \partial_{\varepsilon_{k}} f\left(x^{k}, \cdot\right)\left(x^{k}\right) ; \\
\gamma_{k}:=\max \left\{\lambda_{k},\left\|y^{k}\right\|\right\}, \quad \alpha_{k}:=\frac{\beta_{k}}{\gamma_{k}} ; \\
w^{k}=P_{C}\left(x^{k}-\alpha_{k} y^{k}\right) ; \\
x^{k+1}=\delta_{k} w^{k}+\left(1-\delta_{k}\right) \sum_{i=1}^{p} \eta_{k, i} S_{i}\left(w^{k}\right),
\end{gathered}
$$

for each $k=0,1, \ldots$

Remark 10 (see [10], Remark 2.1). Consider the following:

(i) if $f$ is pseudomonotone on $C$ with respect to the solution set $\operatorname{Sol}(C, f)$ of the problem $\operatorname{EP}(C, f)$, then, under Assumptions 6 (i) and (iv), the set $\operatorname{Sol}(C, f)$ is convex;

(ii) Assumption 6 (ii) is true if whenever Assumption 6 (i) is satisfied and the bifunction $f$ is continuous on $C \times C$; (iii) Assumption 6 (iii) is true if $f$ is pseudomonotone on $C$ and satisfies the paramonotone property as

$$
\begin{gathered}
x \in \operatorname{Sol}(C, f), \quad y \in C, \\
f(y, x)=f(y, x)=0 \Longrightarrow y \in \operatorname{Sol}(C, f) ;
\end{gathered}
$$

(iv) since $f(x, \cdot)$ is lower semicontinuous convex on $C$, applying Remark 5 , we conclude that $\partial_{\varepsilon_{k}} f\left(x^{k}, \cdot\right)\left(x^{k}\right) \neq$ $\emptyset$. Thus, Algorithm 9 is well defined.

Theorem 11. Suppose that Assumptions 6-8 are satisfied. Then, the sequences $\left\{x^{k}\right\}$ and $\left\{w^{k}\right\}$ generated by Algorithm 9 converge strongly to the same point $\bar{x} \in \Omega$, where $\bar{x}=$ $\lim _{k \rightarrow \infty} P_{\Omega}\left(x^{k}\right)$.

Proof. The proof is divided into several steps as follows.

Step 1. For every $x^{*} \in C$ and every $k$, we show that

$$
\left\|x^{k+1}-x^{*}\right\|^{2} \leq\left\|x^{k}-x^{*}\right\|^{2}+2 \alpha_{k} f\left(x^{k}, x^{*}\right)+2 \alpha_{k} \epsilon_{k}+2 \beta_{k}^{2},
$$

and there exists the limit

$$
c:=\lim _{k \rightarrow \infty}\left\|x^{k}-x^{*}\right\| .
$$

For each $k \geq 1$, let

$$
\bar{S}_{k}:=\sum_{i=1}^{p} \eta_{k, i} S_{i} .
$$

By Proposition 2, we see that $\bar{S}_{k}$ is a $\bar{L}$-strict pseudocontraction on $C$ and the sequence $\left\{x^{k}\right\}$ generated by Algorithm 9 can be rewritten as

$$
x^{k+1}:=\delta_{k} w^{k}+\left(1-\delta_{k}\right) \bar{S}_{k}\left(w^{k}\right), \quad \forall k \geq 1 .
$$

Then, for all $k \geq 1$, we have

$$
\begin{aligned}
\| x^{k+1}- & x^{*} \|^{2} \\
= & \left\|\delta_{k} w^{k}+\left(1-\delta_{k}\right) \bar{S}_{k}\left(w^{k}\right)-x^{*}\right\|^{2} \\
= & \left\|\delta_{k}\left(w^{k}-x^{*}\right)+\left(1-\delta_{k}\right)\left(\bar{S}_{k}\left(w^{k}\right)-x^{*}\right)\right\|^{2} \\
= & \delta_{k}\left\|w^{k}-x^{*}\right\|^{2}+\left(1-\delta_{k}\right)\left\|\bar{S}_{k}\left(w^{k}\right)-\bar{S}_{k}\left(x^{*}\right)\right\|^{2} \\
& -\delta_{k}\left(1-\delta_{k}\right)\left\|w^{k}-\bar{S}_{k}\left(w^{k}\right)\right\|^{2} \\
\leq & \delta_{k}\left\|w^{k}-x^{*}\right\|^{2}+\left(1-\delta_{k}\right) \\
& \times\left(\left\|w^{k}-x^{*}\right\|^{2}+\bar{L}^{k}\left(I-\bar{S}_{k}\right)\left(w^{k}\right)-\left(I-\bar{S}_{k}\right)\left(x^{*}\right) \|^{2}\right) \\
& -\delta_{k}\left(1-\delta_{k}\right)\left\|w^{k}-\bar{S}_{k}\left(w^{k}\right)\right\|^{2} \\
= & \left\|w^{k}-x^{*}\right\|^{2}+\left(1-\delta_{k}\right)\left(\bar{L}_{L}\left\|\bar{S}_{k}\left(w^{k}\right)-w^{k}\right\|^{2}\right) \\
& -\delta_{k}\left(1-\delta_{k}\right)\left\|w^{k}-\bar{S}_{k}\left(w^{k}\right)\right\|^{2}
\end{aligned}
$$




$$
\begin{aligned}
= & \left\|w^{k}-x^{*}\right\|^{2}+\left(1-\alpha_{k}\right)\left(\bar{L}-\delta_{k}\right)\left\|\bar{S}_{k}\left(w^{k}\right)-w^{k}\right\|^{2} \\
\leq & \left\|x^{k}-x^{*}\right\|^{2}-\left\|x^{k}-w^{k}\right\|^{2}+2\left\langle x^{k}-w^{k}, x^{*}-w^{k}\right\rangle \\
& +\left(1-\delta_{k}\right)\left(\bar{L}-\delta_{k}\right)\left\|\bar{S}_{k}\left(w^{k}\right)-w^{k}\right\|^{2} \\
\leq & \left\|x^{k}-x^{*}\right\|^{2}-\left\|x^{k}-w^{k}\right\|^{2}+2\left\langle x^{k}-w^{k}, x^{*}-w^{k}\right\rangle \\
\leq & \left\|x^{k}-x^{*}\right\|^{2}+2\left\langle x^{k}-w^{k}, x^{*}-w^{k}\right\rangle .
\end{aligned}
$$

Since $w^{k}=P_{C}\left(x^{k}-\alpha_{k} y^{k}\right)$ and $x^{*} \in C$, we have

$$
\left\langle x^{k}-w^{k}, x^{*}-w^{k}\right\rangle \leq \alpha_{k}\left\langle y^{k}, x^{*}-w^{k}\right\rangle .
$$

Combining this inequality with (30) yields

$$
\begin{aligned}
\left\|x^{k+1}-x^{*}\right\|^{2} \leq & \left\|x^{k}-x^{*}\right\|^{2}+2\left\langle x^{k}-w^{k}, x^{*}-w^{k}\right\rangle \\
\leq & \left\|x^{k}-x^{*}\right\|^{2}+2 \alpha_{k}\left\langle y^{k}, x^{*}-w^{k}\right\rangle \\
= & \left\|x^{k}-x^{*}\right\|^{2}+2 \alpha_{k}\left\langle y^{k}, x^{*}-x^{k}\right\rangle \\
& +2 \alpha_{k}\left\langle y^{k}, x^{k}-w^{k}\right\rangle \\
\leq & \left\|x^{k}-x^{*}\right\|^{2}+2 \alpha_{k}\left\langle y^{k}, x^{*}-x^{k}\right\rangle \\
& +2 \alpha_{k}\left\|y^{k}\right\| x^{k}-w^{k} \| \\
= & \left\|x^{k}-x^{*}\right\|^{2}+2 \alpha_{k}\left\langle y^{k}, x^{*}-x^{k}\right\rangle \\
& +2 \frac{\beta_{k}}{\max \left\{\lambda_{k},\left\|y^{k}\right\|\right\}}\left\|y^{k}\right\|\left\|x^{k}-w^{k}\right\| \\
\leq & \left\|x^{k}-x^{*}\right\|^{2}+2 \alpha_{k}\left\langle y^{k}, x^{*}-x^{k}\right\rangle \\
& +2 \beta_{k}\left\|x^{k}-w^{k}\right\| .
\end{aligned}
$$

Using again $w_{k}=P_{C}\left(x^{k}-\alpha_{k} y^{k}\right)$ and $x^{k} \in C$, we have

$$
\begin{aligned}
\left\|x^{k}-w^{k}\right\|^{2} & \leq \alpha_{k}\left\langle y^{k}, x^{*}-w^{k}\right\rangle \\
& \leq \alpha_{k}\left\|y^{k}\right\|\left\|x^{k}-w^{k}\right\| \\
& =\frac{\beta_{k}}{\max \left\{\lambda_{k},\left\|y^{k}\right\|\right\}}\left\|y^{k}\right\|\left\|x^{k}-w^{k}\right\| \\
& \leq \beta_{k}\left\|x^{k}-w^{k}\right\|,
\end{aligned}
$$

which gives that $\left\|x^{k}-w^{k}\right\| \leq \beta_{k}$. Consequently,

$$
\lim _{k \rightarrow \infty}\left\|x^{k}-w^{k}\right\|=0 \text {. }
$$

This together with (32) implies that

$$
\left\|x^{k+1}-x^{*}\right\|^{2} \leq\left\|x^{k}-x^{*}\right\|^{2}+2 \alpha_{k}\left\langle y^{k}, x^{*}-x^{k}\right\rangle+2 \beta_{k}^{2} \text {. }
$$

Since $y^{k} \in \partial_{\epsilon_{k}} f\left(x^{k}, \cdot\right)\left(x^{k}\right), x^{*} \in C$ and $f(x, x)=0$ for all $x \in C$, we have

$$
\begin{aligned}
\left\langle y^{k}, x^{*}-x^{k}\right\rangle & \leq f\left(x^{k}, x^{*}\right)-f\left(x^{k}, x^{k}\right)+\epsilon_{k} \\
& \leq f\left(x^{k}, x^{*}\right)+\epsilon_{k} .
\end{aligned}
$$

Combining (35) and (36), we obtain that

$$
\begin{aligned}
\left\|x^{k+1}-x^{*}\right\|^{2} \leq & \left\|x^{k}-x^{*}\right\|^{2}+2 \alpha_{k} f\left(x^{k}, x^{*}\right) \\
& +2 \alpha_{k} \epsilon_{k}+2 \beta_{k}^{2} .
\end{aligned}
$$

On the other hand, since $x^{*} \in \operatorname{Sol}(C, f)$, that is, $f\left(x^{*}, x\right) \geq 0$ for all $x \in C$, by pseudomonotonicity of $f$ with respect to $x^{*}$, we have $f\left(x, x^{*}\right) \leq 0$ for all $x \in C$. Replacing $x$ by $x^{k} \in C$, we get $f\left(x^{k}, x^{*}\right) \leq 0$. Then, from (37), it follows that

$$
\left\|x^{k+1}-x^{*}\right\| \leq\left\|x^{k}-x^{*}\right\|^{2}+2 \alpha_{k} \epsilon_{k}+2 \beta_{k}^{2} .
$$

By Assumptions 8 (ii) and (iii), we found that $\sum_{k=1}^{\infty}\left(2 \alpha_{k} \epsilon_{k}+\right.$ $\left.2 \beta_{k}^{2}\right)<\infty$. Using Lemma 3 and (38), we arrive at the existence of

$$
c:=\lim _{k \rightarrow \infty}\left\|x^{k}-x^{*}\right\| .
$$

Step 2. For every $x^{*} \in C$, we show that

$$
\limsup _{k \rightarrow \infty} f\left(x^{k}, x^{*}\right)=0 .
$$

Since $f$ is pseudomonotone on $C$ and $f\left(x^{*}, x^{k}\right) \geq 0$, we have $-f\left(x^{k}, x^{*}\right) \geq 0$. By Step 1 ,

$$
\left\|x^{k+1}-x^{*}\right\|^{2} \leq\left\|x^{k}-x^{*}\right\|^{2}+2 \alpha_{k} f\left(x^{k}, x^{*}\right)+2 \alpha_{k} \epsilon_{k}+2 \beta_{k}^{2} .
$$

We have

$$
\begin{aligned}
2 \alpha_{k}\left[-f\left(x^{k}, x^{*}\right)\right] \leq & \left\|x^{k}-x^{*}\right\|^{2} \\
& -\left\|x^{k+1}-x^{*}\right\|^{2}+2 \alpha_{k} \epsilon_{k}+2 \beta_{k}^{2} .
\end{aligned}
$$

Summing up the above inequalities for every $k$, we obtain that

$$
\begin{aligned}
0 \leq & 2 \sum_{k=0}^{\infty} \alpha_{k}\left[-f\left(x^{k}, x^{*}\right)\right] \leq\left\|x^{0}-x^{*}\right\|^{2} \\
& +2 \sum_{k=0}^{\infty} \alpha_{k} \epsilon_{k}+2 \sum_{k=0}^{\infty} \beta_{k}^{2}<+\infty
\end{aligned}
$$

It follows from the boundedness of the sequences $\left\{y^{k}\right\}$ and $\left\{\lambda_{k}\right\}$ that we can assume that

$$
\max \left\{\lambda_{k},\left\|y^{k}\right\|\right\} \leq M
$$

for a constant $M \geq 0$. Thus,

$$
\alpha_{k}=\frac{\beta_{k}}{\gamma_{k}}=\frac{\beta_{k}}{\max \left\{\lambda_{k},\left\|y^{k}\right\|\right\}} \geq \frac{\beta_{k}}{M},
$$


which together with (43) implies

$$
0 \leq \frac{2}{M} \sum_{k=0}^{\infty} \beta_{k}\left[-f\left(x^{k}, x^{*}\right)\right] \leq 2 \sum_{k=0}^{\infty} \alpha_{k}\left[-f\left(x^{k}, x^{*}\right)\right]<+\infty .
$$

Thus,

$$
\sum_{k=0}^{\infty} \beta_{k}\left[-f\left(x^{k}, x^{*}\right)\right]<+\infty .
$$

Then, by $\sum_{k=0}^{\infty} \beta_{k}=\infty$ and $-f\left(x^{k}, x^{*}\right) \geq 0$, we can deduce that $\lim _{\sup _{k \rightarrow \infty}} f\left(x^{k}, x^{*}\right)=0$ as desired.

Step 3. For any $x^{*} \in \Omega$, suppose that $\left\{x^{k_{j}}\right\}$ is the subsequence of $\left\{x^{k}\right\}$ such that

$$
\limsup _{k \rightarrow \infty} f\left(x^{k}, x^{*}\right)=\lim _{j \rightarrow \infty} f\left(x^{k_{j}}, x^{*}\right),
$$

and, without loss of generality, we may assume that $x^{k_{j}} \rightarrow \bar{x}$ as $j \rightarrow \infty$ for some $\bar{x} \in C$. We show that $\bar{x}$ solves $\operatorname{EP}(C, f)$. To this end, since $f\left(\cdot, x^{*}\right)$ is weakly upper semicontinuous, we have

$$
\begin{aligned}
f\left(\bar{x}, x^{*}\right) & \geq \limsup _{j \rightarrow \infty} f\left(x^{k_{j}}, x^{*}\right)=\lim _{j \rightarrow \infty} f\left(x^{k_{j}}, x^{*}\right) \\
& =\limsup _{j \rightarrow \infty} f\left(x^{k}, x^{*}\right)=0 .
\end{aligned}
$$

On the other hand, since $f$ is pseudomonotone with respect to $x^{*}$ and $f\left(x^{*}, \bar{x}\right) \geq 0$, we have

$$
f\left(\bar{x}, x^{*}\right) \leq 0 .
$$

From (49) and (50), we can conclude that $f\left(\bar{x}, x^{*}\right)=0$. By Assumption 6, we can deduce that $\bar{x}$ is a solution of $E P(f, C)$ as well.

Step 4. We prove that any weakly cluster point of the sequence $\left\{x^{k}\right\}$ is a common fixed point of $L_{i}$-strict pseudocontraction, for each $i=1,2, \ldots, p$. In particular, $\bar{x} \in \bigcap_{i=1}^{p} \operatorname{Fix}\left(S_{i}, C\right)$. Let $\bar{y}$ be any weakly cluster point of $\left\{x^{k}\right\}$ and let $\left\{x^{k_{m}}\right\}$ be a subsequence of $\left\{x^{k}\right\} \subset C$ weakly converging to $\bar{y}$. By convexity and the closedness of $C, C$ is weakly closed. Hence, $\bar{y} \in C$. We first show that

$$
\lim _{m \rightarrow \infty}\left\|x^{k_{m}}-S\left(x^{k_{m}}\right)\right\|=0 .
$$

It follows from (29) that

$$
\begin{aligned}
& \left(1-\delta_{k}\right)\left(\delta_{k}-\bar{L}\right)\left\|\bar{S}_{k}\left(w^{k}\right)-w^{k}\right\|^{2} \\
& \leq\left\|x^{k}-x^{*}\right\|^{2}-\left\|x^{k+1}-x^{*}\right\|^{2}+2 \alpha_{k}\left\langle y^{k}, x^{*}-x^{k}\right\rangle \\
& \quad+2 \beta_{k}\left\|x^{k}-w^{k}\right\| \\
& \leq\left\|x^{k}-x^{*}\right\|^{2}-\left\|x^{k+1}-x^{*}\right\|^{2}+2 \alpha_{k}\left\|y^{k}\right\|\left\|x^{*}-x^{k}\right\| \\
& \quad+2 \beta_{k}\left\|x^{k}-w^{k}\right\|,
\end{aligned}
$$

which gives that

$$
\lim _{k \rightarrow \infty}\left\|\bar{S}_{k}\left(w^{k}\right)-w^{k}\right\|=0
$$

Consequently,

$$
\begin{aligned}
\left\|\bar{S}_{k}\left(w^{k}\right)-x^{k}\right\| \leq & \left\|\bar{S}_{k}\left(w^{k}\right)-w^{k}\right\| \\
& +\left\|x^{k}-w^{k}\right\| \longrightarrow 0 \quad \text { as } k \longrightarrow \infty .
\end{aligned}
$$

By Proposition 2 (i), we arrive at the following:

$$
\begin{aligned}
\left\|\bar{S}_{k}\left(x^{k}\right)-x^{k}\right\| & \leq\left\|\bar{S}_{k}\left(x^{k}\right)-\bar{S}_{k}\left(w^{k}\right)\right\|+\left\|\bar{S}_{k}\left(w^{k}\right)-x^{k}\right\| \\
& \leq \frac{1+\bar{L}}{1-\bar{L}}\left\|x^{k}-w^{k}\right\|+\left\|\bar{S}_{k}\left(w^{k}\right)-x^{k}\right\| \\
& \leq \frac{1+\bar{L}}{1-\bar{L}} \beta_{k}+\left\|\bar{S}_{k}\left(w^{k}\right)-x^{k}\right\| .
\end{aligned}
$$

Thus, we obtain

$$
\lim _{k \rightarrow \infty}\left\|\bar{S}_{k}\left(x^{k}\right)-x^{k}\right\|=0
$$

For each $i=1, \ldots, p$, we suppose that $\left\{\eta_{k_{m}, i}\right\}$ converges to $\eta_{i}$ as $m \rightarrow \infty$ such that $\sum_{i=1}^{p} \eta_{i}=1$. Then, for each $i=1,2, \ldots, p$ and $x \in C$, we have

$$
\begin{aligned}
& \bar{S}_{k_{m}}(x):=\sum_{m=1}^{p} \eta_{k_{m}, i} S_{i}(x) \\
& \longrightarrow \sum_{i=1}^{p} \eta_{i} S_{i}(x):=S(x) \text { as } m \longrightarrow \infty .
\end{aligned}
$$

It follows from (56) that

$$
\begin{aligned}
\left\|x^{k_{m}}-S\left(x^{k_{m}}\right)\right\| \leq & \left\|x^{k_{m}}-\bar{S}_{k_{m}}\left(x^{k_{m}}\right)\right\| \\
& +\left\|\bar{S}_{k_{m}}\left(x^{k_{m}}\right)-S\left(x^{k_{m}}\right)\right\| \\
= & \left\|x^{k_{m}}-\bar{S}_{k_{m}}\left(x^{k_{m}}\right)\right\| \\
& +\left\|\sum_{i=1}^{p} \eta_{k_{m}, S_{i}}\left(x^{k_{m}}\right)-\sum_{i=1}^{p} \eta_{i} S_{i}\left(x^{k_{m}}\right)\right\| \\
= & \left\|x^{k_{m}}-\bar{S}_{k_{m}}\left(x^{k_{m}}\right)\right\| \\
& +\left\|\sum_{i=1}^{p}\left(\eta_{k_{m}, i}-\eta_{i}\right) S_{i}\left(x^{k_{m}}\right)\right\| \\
\leq & \left\|x^{k_{m}}-\bar{S}_{k_{m}}\left(x^{k_{m}}\right)\right\| \\
& +\sum_{i=1}^{p}\left|\eta_{k_{m}, i}-\eta_{i}\right|\left\|S_{i}\left(x^{k_{m}}\right)\right\| .
\end{aligned}
$$


We obtain that

$$
\lim _{j \rightarrow \infty}\left\|x^{k_{m}}-S\left(x^{k_{m}}\right)\right\|=0
$$

By Proposition 2 (ii), we have

$$
\bar{y} \in \operatorname{Fix}(S)=\operatorname{Fix}\left(\sum_{i=1}^{p} \eta_{i} S_{i}\right) x \text {. }
$$

It then follows from Proposition 2 (v) that we have

$$
\bar{y} \in \bigcap_{i=1}^{p} \operatorname{Fix}\left(S_{i}, C\right) .
$$

In particular, we conclude that $\bar{x} \in \bigcap_{i=1}^{p} \operatorname{Fix}\left(S_{i}, C\right)$.

Step 5. Finally, we prove that

$$
\lim _{k \rightarrow \infty} x^{k}=\lim _{k \rightarrow \infty} w^{k}=\lim _{k \rightarrow \infty} P_{\Omega}\left(x^{k}\right)=\bar{x} .
$$

It follows from (38) that, for all $x^{*} \in \Omega$,

$$
\left\|x^{k+1}-x^{*}\right\|^{2} \leq\left\|x^{k}-x^{*}\right\|^{2}+\mu_{k}
$$

where $\mu_{k}:=2 \alpha_{k} \epsilon_{k}+2 \beta_{k}^{2}>0$ for all $k \geq 0$ and $\sum_{k=0}^{\infty} \mu_{k}<+\infty$. Now, using property (14) of the metric projection, we have

$$
\begin{aligned}
&\left\|x^{k+1}-P_{\Omega}\left(x^{k+1}\right)\right\|^{2} \\
&=\left\|\delta_{k}\left(w^{k}-P_{\Omega}\left(x^{k}\right)\right)+\left(1-\delta_{k}\right)\left(\bar{S}_{k}\left(w^{k}\right)-P_{\Omega}\left(x^{k}\right)\right)\right\|^{2} \\
& \leq \delta_{k}\left\|w^{k}-P_{\Omega}\left(x^{k}\right)\right\|^{2}+\left(1-\delta_{k}\right)\left\|\bar{S}_{k}\left(w^{k}\right)-P_{\Omega}\left(x^{k}\right)\right\|^{2} \\
& \leq \delta_{k}\left[\left\|w^{k}-x^{k}\right\|+\left\|x^{k}-P_{\Omega}\left(x^{k}\right)\right\|\right]^{2} \\
&+\left(1-\delta_{k}\right)\left\|\bar{S}_{k}\left(w^{k}\right)-x^{k}\right\|^{2}-\left(1-\delta_{k}\right)\left\|x^{k}-P_{\Omega}\left(x^{k}\right)\right\|^{2} \\
&= \delta_{k}\left\|w^{k}-x^{k}\right\|^{2}+2 \delta_{k}\left\|w^{k}-x^{k}\right\|\left\|x^{k}-P_{\Omega}\left(x^{k}\right)\right\| \\
&+\delta_{k}\left\|x^{k}-P_{\Omega}\left(x^{k}\right)\right\|^{2} \\
&+\left(1-\delta_{k}\right)\left\|\bar{S}_{k}\left(w^{k}\right)-x^{k}\right\|^{2}-\left(1-\delta_{k}\right)\left\|x^{k}-P_{\Omega}\left(x^{k}\right)\right\|^{2} \\
&= \delta_{k}\left\|w^{k}-x^{k}\right\|^{2}+2 \delta_{k}\left\|w^{k}-x^{k}\right\|\left\|x^{k}-P_{\Omega}\left(x^{k}\right)\right\| \\
&+\left(2 \delta_{k}-1\right)\left\|x^{k}-P_{\Omega}\left(x^{k}\right)\right\|^{2}+\left(1-\delta_{k}\right)\left\|\bar{S}_{k}\left(w^{k}\right)-x^{k}\right\|^{2} .
\end{aligned}
$$

Since $\delta_{k} \rightarrow 1 / 2,\left\|w^{k}-x^{k}\right\| \rightarrow 0$, and $\left\|\bar{S}_{k}\left(w^{k}\right)-x^{k}\right\| \rightarrow 0$ as $k \rightarrow \infty$, we obtain that

$$
\lim _{k \rightarrow \infty}\left\|x^{k+1}-P_{\Omega}\left(x^{k+1}\right)\right\|=0
$$

For the simplicity of notation, let $z^{k}:=P_{\Omega}\left(x^{k}\right)$ for each $k \geq 1$. Then, for all $m>k$, since $\Omega$ is convex, we have $(1 / 2)\left(z^{m}+z^{k}\right) \epsilon$ $\Omega$, and therefore

$$
\begin{aligned}
\left\|z^{m}-z^{k}\right\|^{2}= & 2\left\|x^{m}-z^{m}\right\|^{2}+2\left\|x^{m}-z^{k}\right\|^{2} \\
& -4\left\|x^{m}-\frac{1}{2}\left(z^{m}+z^{k}\right)\right\|^{2} \\
\leq & 2\left\|x^{m}-z^{m}\right\|^{2}+2\left\|x^{m}-z^{k}\right\|^{2} \\
& -4\left\|x^{m}-z^{m}\right\|^{2} \\
= & 2\left\|x^{m}-z^{k}\right\|^{2}-2\left\|x^{m}-z^{m}\right\|^{2} .
\end{aligned}
$$

Replacing $x^{*}$ with $z^{k}$ in (63), we can obtain the following:

$$
\begin{aligned}
\left\|x^{m}-z^{k}\right\|^{2} & \leq\left\|x^{m-1}-z^{k}\right\|^{2}+\mu_{m-1} \\
& \leq\left\|x^{m-2}-z^{k}\right\|^{2}+\mu_{m-1}+\mu_{m-2} \\
& \leq \cdots \\
& \leq\left\|x^{k}-z^{k}\right\|^{2}+\sum_{j=k}^{m-1} \mu_{j},
\end{aligned}
$$

Combining this inequality with (66), we have

$$
\left\|z^{m}-z^{k}\right\|^{2} \leq 2\left\|x^{k}-z^{k}\right\|^{2}+2 \sum_{j=k}^{m-1} \mu_{j}-2\left\|x^{m}-z^{m}\right\|^{2},
$$

which gives that

$$
\lim _{m \rightarrow \infty, k \rightarrow \infty}\left\|z^{m}-z^{k}\right\|=0,
$$

which implies also that $\left\{z^{k}\right\}$ is a Cauchy sequence. Hence, $\left\{z^{k}\right\}$ strongly converges to some point $\bar{z} \in \Omega$. However, since $z^{k_{i}}:=$ $P_{\Omega}\left(x^{k_{i}}\right)$, letting $i \rightarrow \infty$, we obtain in the limit that

$$
\bar{z}=\lim _{i \rightarrow \infty} P_{\Omega}\left(x^{k_{i}}\right)=P_{\Omega}(\bar{x})=\bar{x} \in \Omega .
$$

Therefore, $z^{k}:=P_{\Omega}\left(x^{k}\right) \rightarrow \bar{z}=\bar{x} \in \Omega$. Then, from (65), we can conclude that $x^{k} \rightarrow \bar{x}$. Finally, since $\lim _{k \rightarrow \infty}\left\|x^{k}-w^{k}\right\|=$ 0 , we have $\lim _{k \rightarrow \infty} w^{k}=\bar{x}$.

Using Theorem 11, we can obtain the new strong convergence result for finding a common point in the solution set of a class of pseudomonotone equilibrium problems and the set of fixed points of a strict pseudocontraction mapping in a real Hilbert space.

Corollary 12. Let $C$ be a nonempty convex subset of a Hilbert space $\mathscr{H}$. Let the bifunction $f: C \times C \rightarrow \mathbb{R}$ be satisfied by all conditions in Assumption 6. Let $S: C \rightarrow C$ be a L-strict pseudocontraction for some $0 \leq L<1$ such that the solution set $\Omega_{1}$ of the problem $P(C, f, S)$ is nonempty. Suppose that the sequences $\left\{\lambda_{k}\right\},\left\{\beta_{k}\right\},\left\{\varepsilon_{k}\right\}$, and $\left\{\delta_{k}\right\}$ of nonnegative numbers satisfy the following conditions: 
(i) $0<\lambda_{k}<\lambda, 0<L<\delta_{k}<1$, and $\delta_{k} \rightarrow 1 / 2$;

(ii) $\beta_{k}>0, \sum_{k=0}^{\infty} \beta_{k}=+\infty$, and $\sum_{k=0}^{\infty} \beta_{k}^{2}<+\infty$;

(iii) $\sum_{k=0}^{\infty} \beta_{k} \varepsilon_{k}<+\infty$.

Then, the sequence $\left\{x^{k}\right\}$ generated by

$$
\begin{gathered}
x^{0} \in C ; \\
y^{k} \in \partial_{\varepsilon_{k}} f\left(x^{k}, \cdot\right)\left(x^{k}\right) ; \\
\gamma_{k}:=\max \left\{\lambda_{k},\left\|y^{k}\right\|\right\}, \quad \alpha_{k}:=\frac{\beta_{k}}{\gamma_{k}} ; \\
w^{k}=P_{C}\left(x^{k}-\alpha_{k} y^{k}\right) ; \\
x^{k+1}=\delta_{k} w^{k}+\left(1-\delta_{k}\right) S\left(w^{k}\right), \quad k \geq 0
\end{gathered}
$$

converge strongly to a point $\bar{x} \in \Omega_{1}$, where $\bar{x}=$ $\lim _{k \rightarrow \infty} P_{\Omega_{1}}\left(x^{k}\right)$.

Corollary 13. Let $C$ be a nonempty convex subset of a Hilbert space $\mathscr{H}$. Let the bifunction $f: C \times C \rightarrow \mathbb{R}$ be satisfied by all conditions in Assumption 6. Let $S: C \rightarrow C$ be a nonexpansive mapping such that the solution set $\Omega_{2}$ of the problem $P(C, f, S)$ is nonempty. Suppose that the sequences $\left\{\lambda_{k}\right\},\left\{\beta_{k}\right\},\left\{\varepsilon_{k}\right\}$, and $\left\{\delta_{k}\right\}$ of nonnegative numbers satisfy the following conditions:

(i) $0<\lambda_{k}<\lambda, 0<a<\delta_{k}<b<1$, and $\delta_{k} \rightarrow 1 / 2$;

(ii) $\beta_{k}>0, \sum_{k=0}^{\infty} \beta_{k}=+\infty$, and $\sum_{k=0}^{\infty} \beta_{k}^{2}<+\infty$;

(iii) $\sum_{k=0}^{\infty} \beta_{k} \varepsilon_{k}<+\infty$.

Then, the sequences $\left\{x^{k}\right\}$ and $\left\{w^{k}\right\}$ generated by

$$
\begin{gathered}
x^{0} \in C ; \\
y^{k} \in \partial_{\varepsilon_{k}} f\left(x^{k}, \cdot\right)\left(x^{k}\right) ; \\
\gamma_{k}:=\max \left\{\lambda_{k},\left\|y^{k}\right\|\right\}, \quad \alpha_{k}:=\frac{\beta_{k}}{\gamma_{k}} ; \\
w^{k}=P_{C}\left(x^{k}-\alpha_{k} y^{k}\right) ; \\
x^{k+1}=\delta_{k} w^{k}+\left(1-\delta_{k}\right) S\left(w^{k}\right), \quad k \geq 0
\end{gathered}
$$

converge strongly to a point $\bar{x} \in \Omega_{2}$, where $\bar{x}=$ $\lim _{k \rightarrow \infty} P_{\Omega_{2}}\left(x^{k}\right)$.

\section{Conflict of Interests}

The authors declare that there is no conflict of interests regarding the publication of this paper.

\section{Acknowledgment}

The work is supported by Naresuan University.

\section{References}

[1] E. Blum and W. Oettli, "From optimization and variational inequalities to equilibrium problems," The Mathematics Student, vol. 63, no. 1-4, pp. 123-145, 1994.

[2] P. Daniele, F. Giannessi, and A. Maugeri, Equilibrium problems and variational models, Kluwer Academic, Dordrecht, The Netherlands, 2003.

[3] I. V. Konnov, Combined Relaxation Methods for Variational Inequalities, Springer, Berlin, Germany, 2000.

[4] P. N. Anh and J. K. Kim, "An interior proximal cutting hyperplane method for multivalued variational inequalities," Journal of Nonlinear and Convex Analysis, vol. 11, no. 3, pp. 491502, 2010.

[5] P. Ngoc, "A logarithmic quadratic regularization method for pseudomonotone equilibrium problems," Acta Mathematica Vietnamica, vol. 34, no. 2, pp. 183-200, 2009.

[6] H. K. Xu, "An iterative approach to quadratic optimization," Journal of Optimization Theory and Applications, vol. 116, no. 3, pp. 659-678, 2003.

[7] S. Takahashi and W. Takahashi, "Viscosity approximation methods for equilibrium problems and fixed point problems in Hilbert spaces," Journal of Mathematical Analysis and Applications, vol. 331, no. 1, pp. 506-515, 2007.

[8] P. N. Anh, "A hybrid extragradient method extended to fixed point problems and equilibrium problems," Optimization, vol. 62, no. 2, pp. 271-283, 2013.

[9] A. Tada and W. Takahashi, "Weak and strong convergence theorems for a nonexpansive mapping and an equilibrium problem," Journal of Optimization Theory and Applications, vol. 133, no. 3, pp. 359-370, 2007.

[10] P. N. Anh and L. D. Muu, "A hybrid subgradient algorithm for nonexpansive mappings and equilibrium problems," Optimization Letters, vol. 8, no. 2, pp. 727-738, 2014.

[11] K. Goebel and W. A. Kirk, Topics on Metric Fixed Point Theory, Cambridge University Press, Cambridge, UK, 1990.

[12] P. N. Anh, T. D. Quoc, and D. X. Son, "Hybrid extragradienttype methods for finding a common solution of an equilibrium problem and a family of strict pseudo-contraction mappings," Journal of Applied Mathematics and Informatics, no. 3, pp. 13571367, 2012.

[13] G. L. Acedo and H. K. Xu, "Iterative methods for strict pseudocontractions in Hilbert spaces," Nonlinear Analysis: Theory, Methods \& Applications, vol. 67, no. 7, pp. 2258-2271, 2007.

[14] H. Xu, "Viscosity approximation methods for nonexpansive mappings," Journal of Mathematical Analysis and Applications, vol. 298, no. 1, pp. 279-291, 2004.

[15] A. Brøndsted and R. T. Rockafellar, "On the subdifferentiability of convex functions," Proceedings of the American Mathematical Society, vol. 16, pp. 605-611, 1965.

[16] J.-B. Hiriart-Urruty, "Subdifferential calculus," in Convex Analysis and Optimization, pp. 43-92, Pitman, London, UK, 1982.

[17] A. Dhara and J. Dutta, Optimality Conditions in Convex Optimization: A Finite-Dimensional View, Taylor \& Francis Group, LLC, Boca Raton, Fla, USA, 2012. 


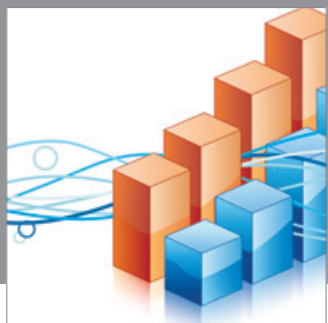

Advances in

Operations Research

mansans

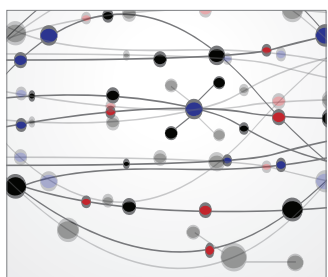

The Scientific World Journal
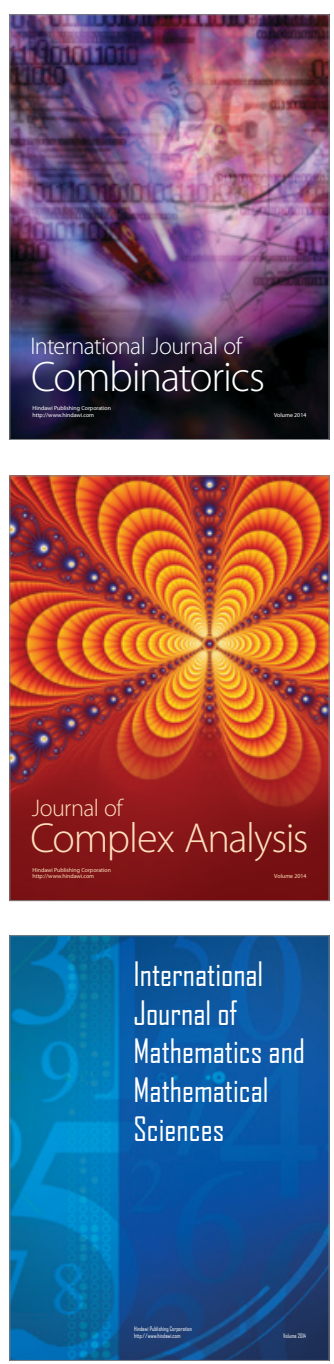
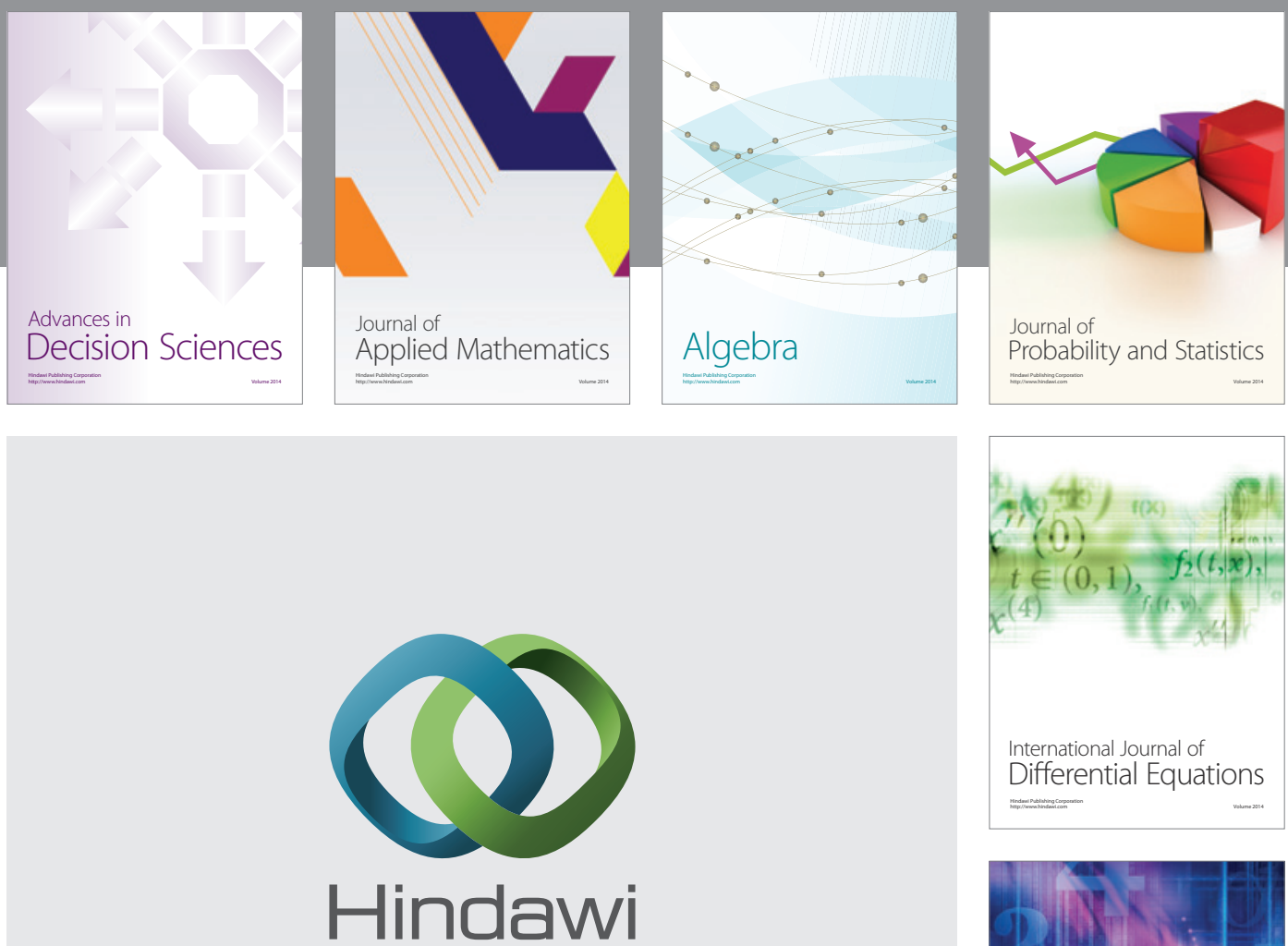

Submit your manuscripts at http://www.hindawi.com
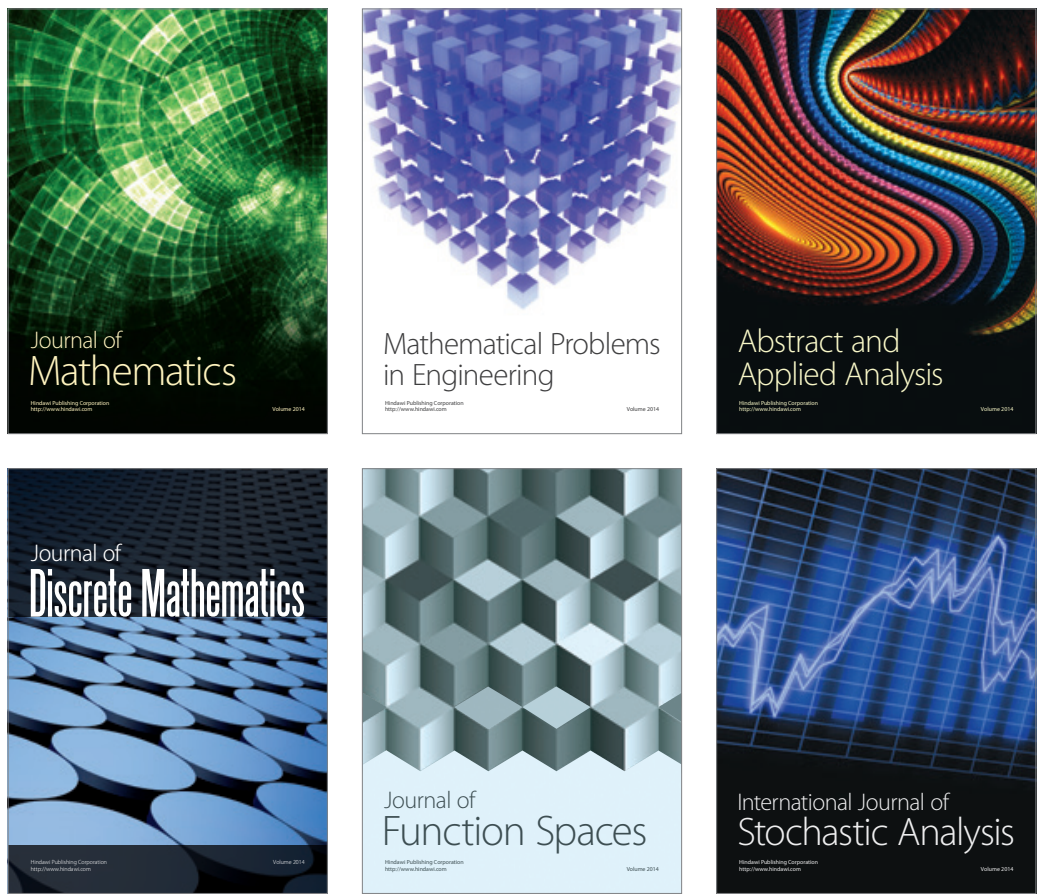

Journal of

Function Spaces

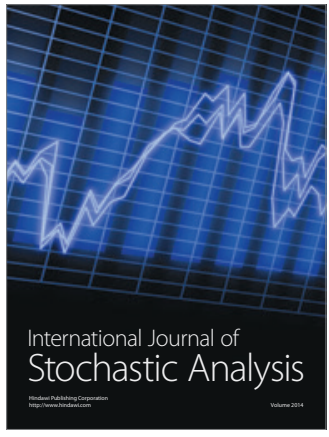

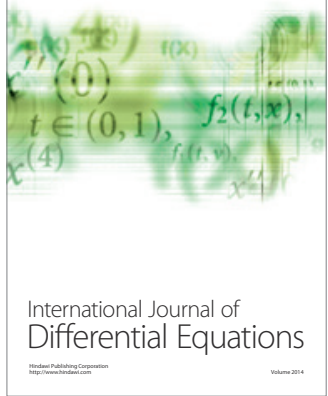
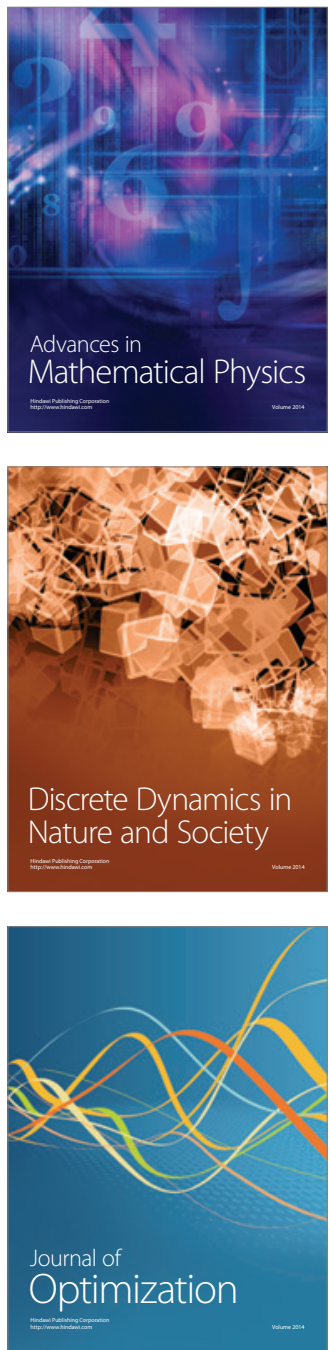